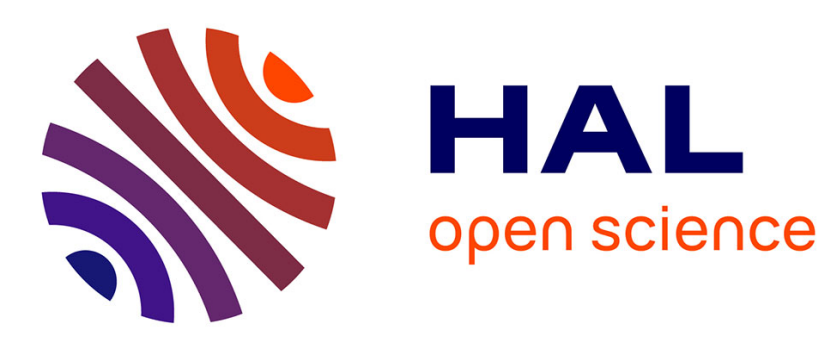

\title{
On the Use of Uniform Circular Arrays for Characterizing UWB Time Reversal \\ Patrice Pajusco, Pascal Pagani
}

\section{To cite this version:}

Patrice Pajusco, Pascal Pagani. On the Use of Uniform Circular Arrays for Characterizing UWB Time Reversal. IEEE Transactions on Antennas and Propagation, 2009, 57 (1), pp.102-109. 10.1109/TAP.2008.2009715 . hal-00688276

\section{HAL Id: hal-00688276 https://hal.science/hal-00688276}

Submitted on 20 Oct 2021

HAL is a multi-disciplinary open access archive for the deposit and dissemination of scientific research documents, whether they are published or not. The documents may come from teaching and research institutions in France or abroad, or from public or private research centers.
L'archive ouverte pluridisciplinaire HAL, est destinée au dépôt et à la diffusion de documents scientifiques de niveau recherche, publiés ou non, émanant des établissements d'enseignement et de recherche français ou étrangers, des laboratoires publics ou privés. 


\title{
On the use of Uniform Circular Array for characterizing UWB Time Reversal
}

\author{
Patrice Pajusco, Member, IEEE and Pascal Pagani, Member, IEEE
}

\begin{abstract}
Time Reversal is a promising technique for the improvement of future telecommunication systems. A better understanding of this capability requires more investigation on real propagation channels. This paper presents a new scheme to characterize the space-time focusing characteristics of time reversal using a Uniform Circular Array. An extensive UWB propagation experiment is reported. The method is applied to the experimental data. Different focusing parameters are computed in different configurations. Because the delay spread parameter is inappropriate, a new parameter called Equivalent Signal to Interference Ratio is introduced to study time focusing.

In UWB SISO configuration, time reversal increases the power of the strongest path by up to $10 \mathrm{~dB}$ and the total received power up to $5 \mathrm{~dB}$.Our analysis shows that when the channel is focused in time, the data rate can by enhanced by a factor of three. The channel is also focused in space and the average size of the spot is about $3 \lambda$ which is larger than the ideal case. The focusing spot is often directed inline with the transmitter. At $9 \lambda$ far from the target, the received signal is reduced by up to $10 \mathrm{~dB}$ in NLOS, which demonstrates the high focusing capabilities of the TRUWB scheme.
\end{abstract}

Index Terms - Circular arrays, Propagation measurements, Time reversal, ultrawideband.

\section{INTRODUCTION}

$\mathrm{T}$ IME REVERSAL (TR) [1] or phase conjugation in frequency domain [2] has been studied for many years. It was initially investigated in the field of acoustics and more recently in the field of electromagnetics. Basically, TR consists in using channel state information at the transmitter. The Channel Impulse Response (CIR) is time reversed and complex conjugated. This corresponds to a matched filter used as a coding waveform. The practical application of TR assumes that the propagation channel is reciprocal and stationary. TR also requires a propagation channel with a large Delay Spread (DS) value. Such channels appear in rich scattering environments.

TR schemes provide spatial and temporal focusing simultaneously at the receiver. Such properties are very promising for communication systems. TR schemes may not

Manuscript received Mars 3, 2008.

P. Pajusco is with with France Telecom / Orange Labs / RESA / NET/ WEP, 6 avenue des Usines, BP 382, 90007 Belfort Cedex, France (e-mail: patrice.pajusco@orange-ftgroup.com).

P. Pagani is with with France Telecom / Orange Labs / RESA / SAFE/ EMC, 2 avenue Pierre Marzin, 22307 Lannion Cedex, France (e-mail: pascal.pagani@orange-ftgroup.com). only increase the offered throughput, but could also reduce the receiver complexity by using non-coherent energy-detection schemes. Furthermore, spatial focusing may enable simultaneous communications between different users, Multiple Input Multiple Output (MIMO) schemes and more secure transmissions.

TR was successfully used in underwater acoustic communication [3]. MIMO transmission schemes were also achieved [4]. Capabilities of TR with electromagnetic waves were investigated in 2002 [5]. Considering channel reciprocity, UWB channel measurements were used to demonstrate the strength of TR. In the same manner, other investigations were done for outdoor mobile wireless access at $2 \mathrm{GHz}$ [6], fixed wireless access at $5 \mathrm{GHz}$ [7] and short range applications [5, 8, 9]. TR of wideband microwaves were experimentally demonstrated in reverberant rooms [10].

UWB and acoustic waves have many similarities. UWB appears to be the most appropriate technology to apply TR because of the success of TR in the field of acoustics. Accurate studies on TR require space-time measurements. Such measurements are usually performed with $2 \mathrm{D}$ virtual planar array antennas. These measurements take a long time and are not often reflected in literature. More data are needed to have relevant statistical results. This paper presents a simple scheme to characterize space-time focusing, based on the use of Uniform Circular Arrays (UCA). A preliminary experimental study using this technique was reported in [11]. In this paper, we significantly expand our analysis by presenting additional measurement results, introducing relevant characteristic parameters and giving theoretical explanations of the observed power gain.

TR is introduced in section II. Then, our innovative characterization method is presented in section III. To illustrate this technique, an UWB propagation experiment is detailed in section IV, and results are analyzed in section V. Finally, conclusions are drawn in section VI.

\section{TIME REVERSAL}

\section{A. Principle}

A radio link is considered between two terminal devices, respectively denoted $\mathrm{A}$ and $\mathrm{B}$. Both of them are assumed stationary. Terminal A is located at the origin of the space. The propagation channel is assumed reciprocal which is true in linear medium. Thus, the CIR is the same regardless of the 
direction of the radio link. Hence, the CIR of the propagation channel between the terminal A and another terminal is expressed by $h(r, \tau)$ where $\tau$ denotes the excess delay and $r$ the location of the terminal. The intended receiver, terminal $\mathrm{B}$, is located at location $r_{0}$. TR consists of using the channel state information at the transmitter. More specifically, the intended receiver B first transmits a pulse, which is sampled at device $A$ to get the CIR $h\left(r_{0}, \tau\right)$. This channel estimate is time reversed and complex conjugated, which is equivalent to a phase conjugation in the frequency domain. This signal is normalized to the unitary power and used as a coding waveform. At any location $r$, the perceived CIR $h_{p e r}(r, \tau)$ will be expressed by equation (1) where $\otimes$ denotes the convolution with respect to the delay $\tau$ and $r_{0}$ the location of the intended receiver.

$$
h_{p e r}(r, \tau)=\frac{h^{*}\left(r_{0},-\tau\right)}{\sqrt{\int\left|h\left(r_{0}, \tau\right)\right|^{2} d \tau}} \otimes h(r, \tau)
$$

It can be noted that the TR scheme is equivalent to a matched filtering performed at the emitter. However, the implementation complexity is significantly reduced with respect to a digital filter.

\section{B. Channel model}

In the next section, selected simulations are presented to illustrate the TR capabilities. A simple propagation channel model is sufficient to highlight the main properties of TR. The Saleh and Valenzuela model [12] was used, with the following parameters : a single cluster, a power decay exponent $\gamma=20 \mathrm{~ns}$, an average ray arrival rate $\lambda=7.5 \mathrm{GHz}$, and a signal bandwidth $\mathrm{BW}=[3.1-10.6 \mathrm{GHz}]$. This configuration provides a CIR with $10 \mathrm{~ns}$ delay spread. This parameter is in accordance with other analyses [13],[14]. Information of direction of arrival was added on each path. Two configurations were considered regarding the distribution of the direction of arrival, the uniform distribution and the laplacian distribution. Lastly, a $20 \log _{10}(f)$ frequency variation was also considered on each path. The above parameters enable the computation of realistic CIRs including space-time characteristics.

\section{Basic simulation results}
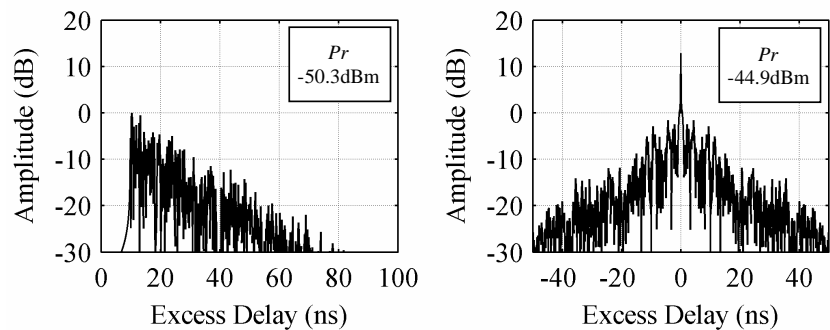

Fig. 1 : Channel model (left) and perceived channel (right)
Figure 1 presents simulation results with $7.5 \mathrm{GHz}$ bandwidth. The instantaneous CIR and the perceived CIR are depicted on the left and the right respectively. The same amplitude scale is used. The perceived channel appears less spread. Using the same transmitted power, the strongest path is significantly increased up to $14 \mathrm{~dB}$ for this specific observation. The total received power is also increased up to $5 \mathrm{~dB}$. This unexpected gain is due to a improved power allocation. By using TR scheme, more power is allocated on less attenuated frequencies. As a result, the transmitted power is less attenuated by the propagation channel resulting in an increase of the received power at the intended receiver.

Let us denote $H(f)$ the channel transfer function, and $P_{T X}$ and $P_{R X}$ the transmitted and received power respectively.

In the case of a simple transmission, the received power $P_{R X}$ can be expressed by equation (2). Considering a TR communication towards the intended receiver, the received power can be expressed by equation (3).

$$
P_{R X}=E\left(\left|\sqrt{P_{T X}} H(f)\right|^{2}\right)=P_{T X} \cdot E\left(|H(f)|^{2}\right)
$$

where $E$ denotes the mathematical expectation.

$$
\begin{aligned}
P_{R X_{-} R T} & =E\left(\left|\sqrt{P_{T X}} H(f) \cdot \frac{H^{*}(f)}{E\left(|H(f)|^{2}\right)}\right|^{2}\right) \\
& =P_{R X} \cdot \frac{E\left(|H(f)|^{4}\right)}{E\left(|H(f)|^{2}\right)^{2}}
\end{aligned}
$$

Let us now consider the statistical distribution of the magnitude of the transfer function $|H(f)|$, and denote $\mu_{2}=E\left(|H(f)|^{2}\right)$ and $\mu_{4}=E\left(|H(f)|^{4}\right)$ its second order and fourth order raw moments respectively. Equation (3) shows that the power gain of the TR scheme is given by the ratio $\mu_{4} /\left(\mu_{2}\right)^{2}$. In a rich scattering indoor environment, $H(f)$ can be modeled by a complex Gaussian distribution, and its magnitude $|H(f)|$ thus follows a Rayleigh distribution with parameter $\sigma$. In this case, the TR power gain is given by:

$$
\frac{\mu_{4}}{\left(\mu_{2}\right)^{2}}=\frac{8 \sigma^{4}}{\left(2 \sigma^{2}\right)^{2}}=2
$$

This result highlights that $3 \mathrm{~dB}$ gain is achieved when TR scheme is used in a rich scattering environment. Interestingly, if the radio link consists of a strong main path and some weaker random components, it can be assumed that $|H(f)|$ follows a Rice distribution with parameter $k$. In this case, it can be shown that the TR power gain is given by:

$$
\frac{\mu_{4}}{\left(\mu_{2}\right)^{2}}=2-\frac{k^{2}}{k^{2}+2 k+1}
$$


The highest gain is obtained for $k=0$ (Rayleigh distribution), while for a high value of $k$ no gain is achieved, since a single echo is predominant resulting in a flat and nearly constant channel transfer function.
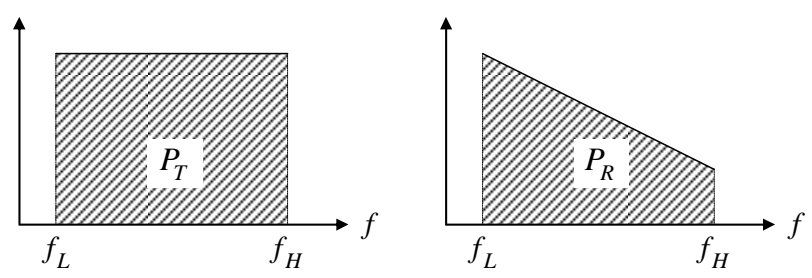

Fig. 2 : Power density of the transmitted and received power

When a large bandwidth is used, the effect of frequency can no longer be ignored. A simple channel model with a $20 \log _{10}(f)$ attenuation is considered. This frequency slope corresponds to the typical case found in our previous results [15]. The left of the Fig. 2 denotes the spectrum of an UWB signal. $f_{L}, f_{H}$ and $P_{T}$ denote the lowest frequency, the highest frequency and the total transmitted power respectively. The power density of the transmitted signal is given by equation (6).

$$
\left|v_{t x}(f)\right|=\sqrt{\frac{P_{T X}}{f_{H}-f_{L}}}
$$

The right of the Fig. 2 depicts the spectrum of the received signal where $P_{R X}$ denotes the total received power. All these assumptions are expressed by equations 7-8.

$$
\begin{gathered}
|H(f)|=\frac{k}{f} \\
P_{R X}=\int_{f_{L}}^{f_{H}}\left|h(f) \cdot v_{t x}(f)\right|^{2} d f=P_{T X} \frac{k^{2}}{f_{L} \cdot f_{H}}
\end{gathered}
$$

If a TR scheme is considered, the spectrum of the transmitted signal $v_{t x_{-} R T}(f)$ is the same of the received signal. The same transmitted power is assumed. The new transmitter signal and received power are expressed by equation 9 and 10 respectively.

$$
\begin{gathered}
\left|v_{t x_{-} T R}(f)\right|=\sqrt{\frac{f_{L} \cdot f_{H}}{f_{H}-f_{L}}} \cdot \frac{\sqrt{P_{T X}}}{f} \\
P_{R x_{-} T R}=P_{T X} \frac{k^{2}}{3 f_{L} \cdot f_{H}}\left(1+\frac{f_{L}}{f_{H}}+\frac{f_{H}}{f_{L}}\right)
\end{gathered}
$$

The two previous gains are independent. The comprehensive gain of power can be approximated by adding these previous gains. The TR gain of the total is given by equation (11). This corresponds to a $5 \mathrm{~dB}$ gain with $7.5 \mathrm{GHz}$ bandwidth.

$$
\Gamma(d B)=-1.76+10 \log _{10}\left(1+\frac{f_{L}}{f_{H}}+\frac{f_{H}}{f_{L}}\right)
$$
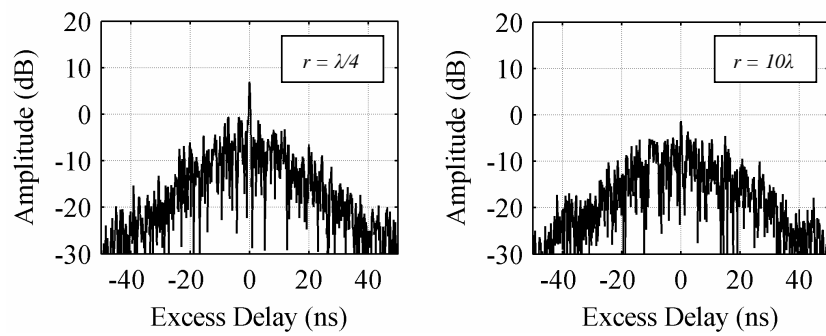

Fig. 3 : Influence of the location on the perceived CIR

At the intended receiver depicted in Fig. 1 (right), the peak level is situated at $14 \mathrm{~dB}$ above the side lobes. CIRs and perceived CIRs were computed at different locations to investigate spatial behavior of TR. The perceived CIRs at two different locations away from the intended receiver are depicted in Fig. 3. When the receiver moves away from the target, the main path amplitude decreases and the shape is no longer symmetrical. At $\lambda / 4$ (left), the peak is $6 \mathrm{~dB}$ below the maximum. At $10 \lambda$ (right), the peak is completely lost in the side lobes. This underlines the capability of TR to focus the signal in space.

The space focusing capability can also be plotted in two dimensions to analyze the shape and the size of the focusing area. The maximum amplitude of the perceived CIR at each received location is represented in Fig. 4. The intended receiver was chosen at the center of the plane. The characteristics of the spot area remain almost the same when the intended receiver is moved. On the left of Fig. 4, the results were obtained with a uniform distribution of direction of arrival of the rays. It corresponds to a rich scattering environment. The spot is circular and the size is about $\lambda / 2$ as previously measured [10]. On the right of Fig. 4, the simulation was performed with an angular spread of $20^{\circ}$. It is more representative of a real environment. The spot is no longer circular and is directed inline with the transmitter. These two examples highlight the fact that the directional properties of the propagation channel are very important for spatial focusing capability.
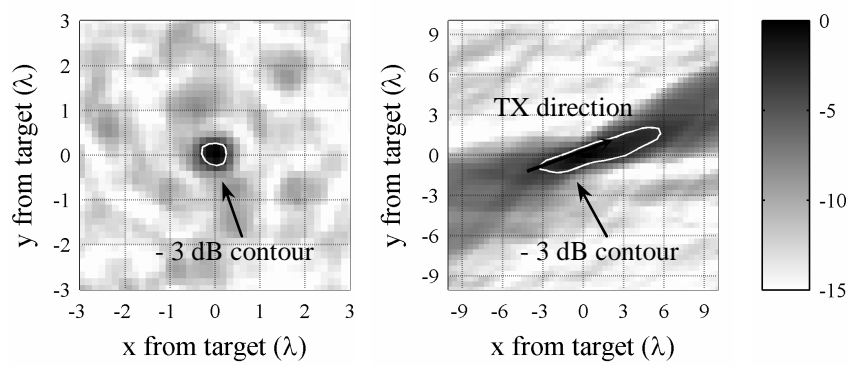

Fig. 4 Focusing area with uniform distribution and laplacian $\left(\mathrm{AS}=20^{\circ}\right)$ distribution of angles of arrival 


\section{Synthetic parameters}

In this section, we present a number of synthetic parameters characterizing the temporal and spatial focusing properties of TR.

\section{1) Delay spread}

The Delay Spread (DS) parameter is widely used to study wideband channels. It is often related to the achievable data rates. It is computed with the second moment of the average power delay profile. This parameter characterizes the temporal spreading of the CIR. A Hanning window is used to reduce sidelobes and compute the DS value. Table 1 shows the results for different threshold values using the channel model depicted in Fig. 1.

TABLE I

INFLUENCE OF THRESHOLD ON DELAY SPREAD VALUES

\begin{tabular}{ccc}
\hline \hline \multirow{2}{*}{$\begin{array}{c}\text { Threshold } \\
(\mathrm{dB})\end{array}$} & \multicolumn{2}{c}{ Delay Spread (ns) } \\
\cline { 2 - 3 } & Channel & TR channel \\
\hline 15 & 7.1 & 0.1 \\
20 & 8.0 & 3.2 \\
30 & 9.4 & 8.0 \\
40 & 9.8 & 9.6 \\
50 & 9.9 & 10.0 \\
\hline \hline
\end{tabular}

This highlights that the DS parameter is very sensitive to the noise cut-off threshold. For example, TR can decrease or increase the DS value depending on the cut-off level. In fact, with this simple channel model, the DS value is not affected by the TR scheme if the noise cut-off level is taken sufficiently low. Such a parameter is not appropriate to qualify time focusing of TR. To avoid biased results, a new parameter is introduced in the next section.

2) Equivalent Signal to Interference Ratio

In a practical communication system, when the Signal to Noise Ratio (SNR) is sufficiently high, the achievable Bit Error Rate (BER) is only limited by the Inter Symbol Interference (ISI). ISI is generated by the dispersive nature of the propagation channel, where the desired signal is corrupted at the receiver by echoes of previously transmitted symbols. In a noise-free configuration, and assuming a simple receiver implementation with no specific equalization technique, the average ISI can be evaluated knowing the symbol rate and the CIR. It can be assumed that the receiver supports the ability to synchronize with the strongest path in the CIR. For instance, this would be a simple one finger RAKE receiver. In such a system, the useful signal power $S$ is given by :

$$
S=\left|h\left(\tau_{\max }\right)\right|^{2}
$$

where $h(\tau)$ represents the CIR and $\tau_{\max }$ is the delay of the strongest path in the CIR. Similarly, assuming that transmitted symbols are independent and that multi-path interference is equivalent to a Gaussian noise, the interference power $I$ is given by :

$$
I=\left.\sum_{\substack{k=-\infty \\ k \neq 0}}^{k=+\infty} h\left(\tau_{\max }+k / R\right)\right|^{2}
$$

where $R$ denotes the system symbol rate.

In order to characterize the improvement of TR accurately, we introduce a new parameter called Equivalent Signal to Interference Ratio (ESIR). It corresponds to the average SIR for a given symbol rate $\mathrm{R}$. This parameter is defined in $\mathrm{dB}$ by :

$$
\operatorname{ESIR}(R)=10 \log _{10}\left(\frac{\left|h\left(\tau_{\max }\right)\right|^{2}}{\sum_{\substack{k=-\infty \\ k \neq 0}}^{k=+\infty}\left|h\left(\tau_{\max }+k / R\right)\right|^{2}}\right)
$$

Based on this parameter, an estimate of the maximal symbol rate to achieve a given ESIR of $x \mathrm{~dB}$ in a noise free configuration is $R_{x d B}$ such as :

$$
\operatorname{ESIR}\left(R_{x_{d B}}\right)=x
$$

Considering a static channel and a simple uncoded BPSK modulation scheme, a SIR of $10.5 \mathrm{~dB}$ is required to achieve a BER of $10^{-6}$ [16]. Hereunder, the parameter $R_{10.5}$ will be computed to evaluate the maximum bit rate of a basic receiver structure. The variation of this parameter when applying a TR scheme will give accurate insights to the benefits of TRcapable systems.

\section{3) Temporal peak to total energy ratio}

This quantity corresponds to the ratio between the power of the strongest path and the total power. It characterizes the ratio of energy captured by a simple receiver

$$
\vartheta(h)=10 \log _{10}\left(\frac{\max _{\tau}\left(|h(r, \tau)|^{2}\right)}{\int\left|h\left(r_{0}, \tau\right)\right|^{2} d \tau}\right)
$$

\section{4) Peak to Peak Gain}

A simple receiver structure estimates data by synchronizing with the strongest path of the CIR and sampling the received signal. In order to study the improvement of TR, the Peak to Peak gain parameter $p 2 p(h)$ is used. This parameter corresponds to the ratio between the strongest path of the CIR and the strongest path of the perceived CIR. It is defined by:

$$
p 2 p(h)=10 \log _{10}\left(\frac{\max _{\tau}\left(\left|h_{p e r}\left(r_{0}, \tau\right)\right|^{2}\right)}{\max _{\tau}\left(\left|h\left(r_{0}, \tau\right)\right|^{2}\right)}\right)
$$

where $r_{0}$ denotes the target location

5) Total received power gain

Advanced receiver structures, such as RAKE receivers with a large number of fingers, are able to capture all the power carried by the CIR. In order to evaluate potential gain of TR, the total received power gain $\Gamma$ is defined by: 


$$
\Gamma(h)=10 \log _{10}\left(\frac{\int\left|h_{p e r}\left(r_{0}, \tau\right)\right|^{2} d \tau}{\int\left|h\left(r_{0}, \tau\right)\right|^{2} d \tau}\right)
$$

\section{6) Spatial focusing parameter}

Using spatial focusing, a TR-capable communication system may transmit streams simultaneously towards nearby users. More precisely, TR may focus the signal enough in space so that different nearby users may enjoy two different communication channels with low mutual interference. To evaluate interference between users, the spatial focusing gain parameter is defined by:

$$
\eta(r)=10 \log _{10}\left(\frac{\max _{\tau}\left(\left|h_{p e r}(r, \tau)\right|^{2}\right)}{\max _{\tau}\left(\left|h_{p e r}\left(r_{0}, \tau\right)\right|^{2}\right)}\right)
$$

\section{ON THE USE OF UWB CIRCULAR MEASUREMENTS}

Space-time measurements are required to characterize TR accurately. A virtual planar array of antennas is one way to sample the propagation channel in space. The first study on TR using microwaves adopted this approach [5]. However, such an experiment requires a long time. To cover the area, a large number of locations are needed. Moreover, it requires a precise $\mathrm{X}-\mathrm{Y}$ positioning device. Usually, a simple linear array is considered in TR studies and experiments. The main drawback is that only one direction is spatially sampled. This approach can introduce biased results because the focusing area is not symmetric in practice. Uniform Circular Array (UCA) measurements can solve planar and linear measurement drawbacks. In the following, we will show that an UCA configuration offers an accurate $2 \mathrm{D}$ scanning of the TR effects while maintaining a reasonable measurement time. The configuration of the UCA is depicted in Fig. 5. Each location is denoted $L_{k}$, where $k$ is the $k^{\text {th }}$ sensor. Locations are uniformly distributed on the circle. $\mathrm{N}$ is the number of sensors and is assumed even. $d$ is the diameter of the circle.

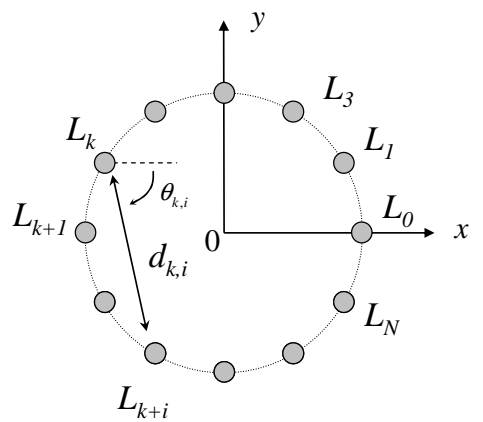

$$
\begin{aligned}
& \left|\overrightarrow{L_{k} L_{k+i}}\right|=d \cdot \sin \left(\frac{\pi i}{N}\right)=d_{i} \\
& \theta_{k, i}=\theta_{k, 1}+\frac{\pi i}{2 N} \\
& \text { with } i \in\left[0, \frac{N}{2}\right]
\end{aligned}
$$

Fig. 5: Configuration of the circular array

The distance $\left|\overrightarrow{L_{k} L_{k+i}}\right|$ depends only on $i$ and is denoted $d_{i}$. It ranges from $d_{1}$ to $d_{N / 2}$, which respectively correspond to the smallest spacing between two sensors and the diameter. $\theta_{k, i}$ denotes the direction of $\overrightarrow{L_{k} L_{k+i}}$ relatively to the $0 \mathrm{x}$ axis. For each value of $d_{i}, \mathrm{~N} / 2$ values of $\theta_{k, i}$ are uniformly distributed in the range [0 $2 \pi]$.

Now, $L_{k}$ is assumed a target of a TR transmission and $L_{k+i}$ a location of interest. According to previous comments, the space-time focusing gain at different distances and/or in different directions can be computed. For instance, the average focusing gain can be derived at different distances between 0 and $d$. The 2D space time focusing can also be computed. This principle was applied to the two previous channel models and the results are plotted in Fig. 6. Even if the input data are smaller, the shape and amplitude are the same as the reference in Fig. 4.
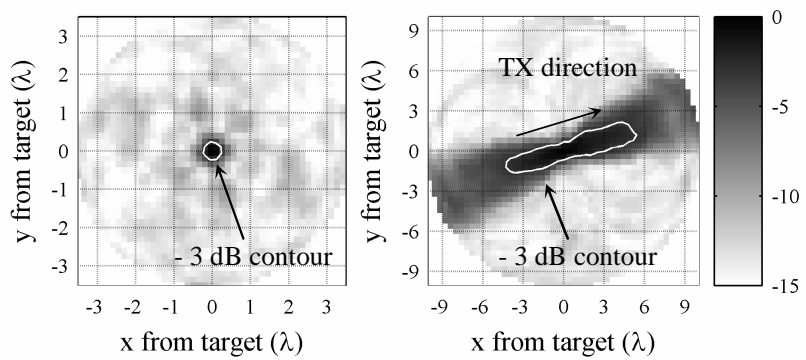

Fig. 6: Focusing area with uniform distribution and laplacian $\left(\mathrm{AS}=20^{\circ}\right)$ distribution of angles of arrival, using UCA simulations

To conclude this section, measurements using UCA are an efficient way to investigate TR. The positioning system is simple. This configuration provides many combinations of angles and distances from the target. It enables reliable computation of the focusing gain parameters. The 2D focusing area can be also obtained.

\section{UWB PROPAGATION MEASUREMENT}

The UWB propagation channel measurements described in this section were previously presented in [17]. For the purpose of this study, the main characteristics of the experiment are recalled. The CIRs were obtained using standard $\mathrm{S}_{21}$ parameter measurements. The schematic of the measurement device is depicted in Fig. 7.

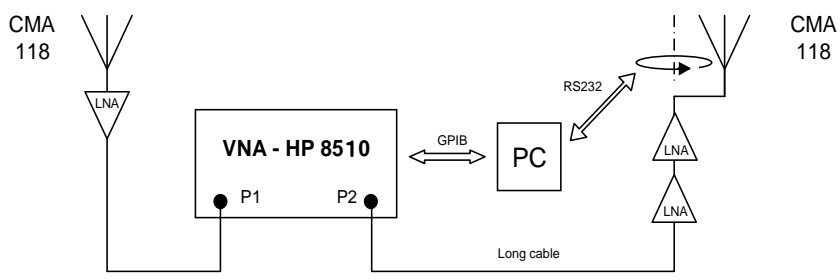

Fig. 7 : Schematic diagram of the measurement device

The propagation channel was sampled over 4005 equally spaced frequency tones between 3.1 and $11.1 \mathrm{GHz}$. Two indoor office configurations were investigated. The distance between transmitter and receiver ranges from 1 meter to 18 meters. For each transmitted location, the channel was probed at 90 regularly spaced positions along a circle of $40 \mathrm{~cm}$ in 
diameter. More than 120 locations were measured corresponding to just under 11000 UWB CIRs.

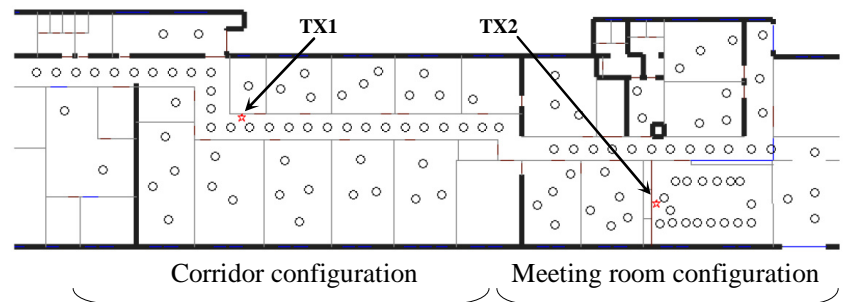

Fig. 8 Floor plan of office building where the UWB measurements took place

\section{StatisticAl ANALYSIS}

\section{A. Statistical results}

For each measurement location, 90x90 combinations are available to compute focusing parameters. Thus, about 1 million pairs of links were evaluated. All parameters were computed for different bandwidths. The average results for the largest bandwidth in LOS and NLOS situations are reported in Table II.

In the NLOS configuration TR increases the power captured by the strongest path. The amplitude of the strongest path is about $12 \mathrm{~dB}$ above the amplitude of the strongest path of the propagation channel. This illustrates the time focusing efficiency of TR and induces a significant improvement of coverage if simple receivers are used. Nevertheless, the DS remains unchanged at the intended receiver. It was also observed with a smaller frequency bandwidth [7]. Only the MISO configuration is able to reduce the delay spread. Nevertheless, the data rate can be increased by a factor of three according to the new parameter introduced in section II.B. In LOS situation, these trends are the same but improvements are smaller.

TABLE II

STATISTICAL RESULTS OF FOCUSING GAIN PARAMETERS (7.5GHZ BANDWIDTH, AVERAGE VALUES)

\begin{tabular}{ccc}
\hline \hline Configuration & LOS & NLOS \\
\hline$\vartheta_{C I R}$ & $29 \%$ & $8.7 \%$ \\
$\vartheta_{T R}$ & $34 \%$ & $22 \%$ \\
$D S_{C I R}$ & $8.8 \mathrm{~ns}$ & $17 \mathrm{~ns}$ \\
$D S_{T R}\left(r_{0}\right)$ & $9.5 \mathrm{~ns}$ & $18 \mathrm{~ns}$ \\
$\Gamma$ & $5.1 \mathrm{~dB}$ & $7 \mathrm{~dB}$ \\
$R_{10.5}^{R T} / R_{10.5}^{C I R}$ & 1.2 & 3.1 \\
$n(9 \lambda)$ & $-5 \mathrm{~dB}$ & $-10 \mathrm{~dB}$ \\
$p 2 p$ & $6 \mathrm{~dB}$ & $12 \mathrm{~dB}$ \\
\hline \hline
\end{tabular}
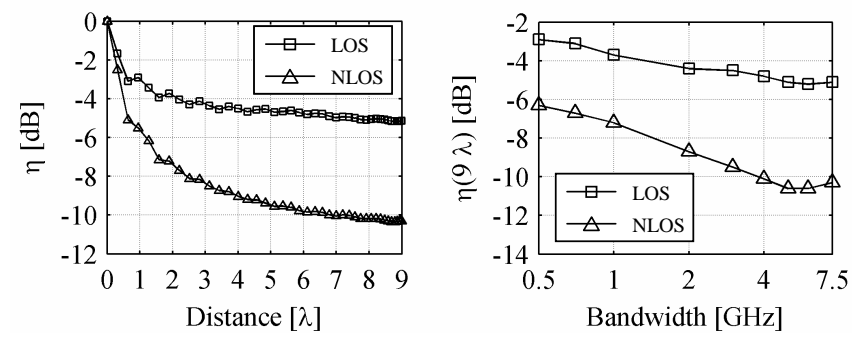

Fig. 9 : Statistical results of space focusing in LOS/NLOS configuration
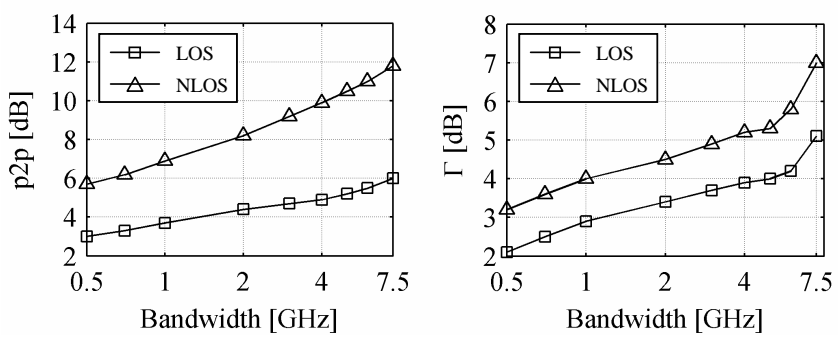

Fig. 10 : Statistical results of power gain in LOS/NLOS configuration

The average focusing gain $\eta(r)$ is depicted in Fig. 9 (left). LOS and NLOS situations are shown. The space focusing is more efficient in the NLOS situation. In NLOS the average size of the focusing area (measured at the $-6 \mathrm{~dB}$ level) is about $3 \lambda$ which is higher than the ideal case, corresponding to a uniform distribution of angles of arrival. The main explanation is that there is usually a dominant direction in indoor environments. This direction is usually the direction of the transmitter. Thus the space focusing gain is much lower in the direction of the transmitter. In the LOS situation, the focusing gain does not reach $-6 \mathrm{~dB}$. On the right of Fig. 9, the influence of bandwidth is presented. The bandwidth is represented on a $\log$ scale. The greater the bandwidth, the higher the spatial focusing gain.

Fig. 10 depicts the gain of the main peak power for different bandwidths. An acceptable linear dependence is found. The slope is higher in the NLOS than in the LOS situation. The gain in the total received power is a little higher than the simulation. A possible explanation is the basic channel model used in simulation and the effects of antenna.

\section{B. Typical results}

According to the statistical analysis and a visual inspection of all the space-time focusing diagrams, a typical file was selected, the parameters of which are close to the mean values. The main results are reported in Fig. 11. This example illustrates the previous trends found in the statistical analysis. On the left, a gain of $15 \mathrm{~dB}$ can be observed on the strongest path of the two power delay profiles. On the right, the focusing area is elongated inline with the transmitter. The smaller values are obtained orthogonally to the direction of the transmitter. 

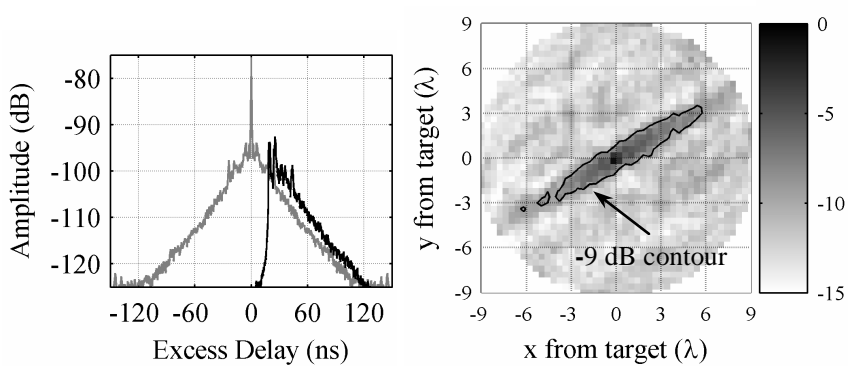

Fig. 11: Typical measurement results in indoor environment

\section{CONCLUSIONS}

This paper presents an efficient method of characterizing the space-time focusing gain of SISO TR. It was verified with a propagation channel model and applied to UWB measurements. Focusing parameters of a typical indoor office environment were obtained. A $10 \mathrm{~dB}$ gain can be achieved on the strongest path using the entire FCC UWB bandwidth. The gain can provide significant improvement in coverage when simple receivers are used. SISO TR does not change the DS value. Nevertheless, the data rate can be increased in NLOS by a factor of three according to a new introduced parameter. The average size of the focusing area is about $3 \lambda$ but is strongly dependant on the observation direction. This is probably not small enough to enable simultaneous streams towards the same user, but very promising for the reduction of interference between UWB users.

\section{REFERENCES}

[1] A. Derode, P. Roux, and M. Fink, "Acoustic time-reversal through high-order multiple scaterring," presented at IEEE Ultrasonics Symposium, Seattle, WA, USA, Nov. 1995.

[2] D. R. Jackson and D. R. Dowling, "Phase conjugation in underwater acoustics," Journal of the Acoustical Society of America, vol. 89, pp. 171-181, Jan. 1991.

[3] G. F. Edelmann, T. Akal, W. S. Hodgkiss, S. Kim, W. A. Kupperman, and H. C. Song, "An initial demonstration of underwater acoustic communication using time reversal," IEEE J. Ocean. Eng., vol. 27, July 2002.

[4] H. C. Song, W. S. Hodgkiss, P. Roux, W. A. Kuperman, T. Akal, and M. Stevenson, "Coherent MIMO time reversal communications in shallow water," presented at OCEANS 2004, Kobe, Japan, Nov. 2004.

[5] S. M. Emami, J. Hansen, A. D. Kim, G. Papanicolaou, A. J. Paulraj, D. Cheung, and C. Prettie, "Predicted time reversal performance in wireless communication using channel measurements," IEEE communication letters, vol. XX, 2002.

[6] H. T. Nguyen, J. B. Andersen, and G. F. Pedersen, "The potential use of time reversal techniques in multiple element antenna systems," IEEE communication letters, vol. 9, pp. 40-42, Jan. 2005.

[7] P. Kyritsi, G. Papanicolaou, P. Eggers, and A. Oprea, "Time reversal techniques for wireless communications," presented at Vehicular Technology Conference, Sept. 2004.

[8] A. E. Akogun, C. R. Qiu, and N. Guo, "Demonstrating Time Reversal in Ultra-wideband Communications using Time Domain Measurements," presented at International Instrumentation Symposium, Knoxville, Tennessee, May 2005.
[9] R. C. Qiu, C. J. Zhou, N. Guo, and J. Q. Zhang, "Time reversal with MISO for ultrawideband communications: experimental results," Antennas and Wireless Propagation Letters, vol. 5, pp. 269, Dec. 2006.

[10] G. Lerosey, J. de Rosny, A. Tourin, A. Derode, and M. Fink, "Time reversal of wideband microwaves," Applied Physics Letters (154101), 2006.

[11] P. Pajusco and P. Pagani, "Characterization of UWB Time Reversal using circular array measurements," ECWT, 2007.

[12] A. Saleh and R. Valenzuela, "A Statistical Model for Indoor Multipath Propagation," IEEE J. Sel. Areas Commun., vol. 5, pp. 128-137, Feb. 1987.

[13] R. M. Buehrer, W. A. Davis, A. Safaai-Jazi, and D. Sweeney, "Characterization of the ultra-wideband channel," presented at IEEE Conference on Ultra Wideband Systems and Technologies, Reston VA, USA, Nov. 2003.

[14] S. S. Ghassemzadeh, L. J. Greenstein, A. Kavcic, T. Sveinsson, and V. Tarokh, "UWB indoor delay profile model for residential and commercial environments," presented at IEEE Vehicular Technology Conference, Orlando, FL, USA, Oct. 2003.

[15] P. Pajusco and P. Pagani, "Frequency Dependence of The UWB Indoor Propagation Channel," presented at Eucap, Edinbourg, 2007.

[16] J. G. Proakis, Digital Communications, McGraw-Hill 4th ed. New York, 2000.

[17] P. Pagani and P. Pajusco, "Experimental Analysis of the Ultra Wideband Propagation Channel over the $3.1 \mathrm{GHz}-10.6 \mathrm{GHz}$ Frequency Band," presented at PIMRC, Helsinki, Sept. 2006.

Biographies to be provided later. 